\title{
Pediatric procedures in urology residency training: An analysis of the experience of Canadian urology residents
}

\author{
Paul Gustafson, MD; Andrew MacNeily, MD, FRCSC
}

Department of Urologic Sciences, University of British Columbia, Vancouver, BC

See related article on page 321.

Cite as: Can Urol Assoc J 2014;8(9-10):317-20. http://dx.doi.org/10.5489/cuai.2004

Published online October 13, 2014.

\section{Abstract}

Introduction: We sought to determine if the exposure to pediatric urologic procedures by graduates of Canadian urological programs is congruent with the objectives of training (OTR) put forward by the Royal College of Physicians and Surgeons of Canada (RCPSC). Methods: The Canadian T-Res (Resiliance Software Inc., Vancouver, BC) database for pediatric surgical procedures logged from 2003 to 2009 was interrogated. The number of cases logged for each of the A, B and C lists of procedures (least complex to most complex) as outlined in the RCPSC OTR in Urology were recorded for the 6 participating programs across the country.

Results: A total of 48 residents submitted data to T-Res from the 6 participating programs. Of the A-list procedures, Canadian urology residents (PGY 1-5) from the 6 participating programs participated in an annual average of 53 hypospadias repairs, 30 orchidopexies for inguinal testes, 26 circumcisions, 7 hernia/hydrocele repairs, 7 pyeloplasties, 7 ureteral reimplants, 6 endoscopic injections for vesicoureteral reflux, 3 meatoplasties/meatotomies, 1 transurethral incision of ureterocele, 2 endoscopic procedures for stone management, and 1 transurethral incision of ureterocele, during the years in question. Of the B-list procedures, residents participated in an annual average of 1 transurethral resection of a posterior urethral valve, 3 continent diversions, 2 augmentation cystoplasties and 1 vesicostomy. Of the data available for the C-list procedures, residents participated in an annual average of less than 1 exstrophy repair and less than 1 pediatric renal transplant.

Conclusions: The RCPSC objectives set out by the specialty committee are a useful framework for guiding graduating residents on which procedures they might reasonably perform once they enter practice. Ongoing revisions to these objectives, which reflect changing trends in the management of core pediatric urology procedures, are supported by our study based on the number of cases in which residents participate. Improvements in the assessment of trainee surgical experience and competence, as it relates to the objectives of training in pediatric urology, are required as we migrate towards a competency-based model of postgraduate medical education.

\section{Introduction}

Pediatric urology has become increasingly subspecialized. In addition to post-residency fellowship training, urologists can now also acquire a certificate of added qualification (CAQ) that requires successful completion of an examination administered by the American Board of Urology., Nevertheless, pediatric urology remains a core component of urological residency training in North America. ${ }^{3}$

A previous study assessed the perceived competence of Canadian urology residents in performing pediatric urological procedures upon graduation from residency. ${ }^{4}$ This study focused on which pediatric urological procedures Canadian residents, program directors and practicing pediatric urologists felt graduates should be competent to perform, and which they would actually perform, without further fellowship training.

Our goal was to expand upon this study by assessing the volume and scope of pediatric urological procedures in which Canadian residents participate. Moreover, we aimed to determine if the exposure to pediatric urologic procedures by graduates of Canadian urological programs is congruent with the objectives of training (OTR) put forward by the Royal College of Physicians and Surgeons of Canada (RCPSC).

\section{Methods}

After obtaining permission from the Specialty Committee in Urology, we consulted the Canadian T-Res (Resiliance Software Inc., Vancouver, BC) database for pediatric surgical procedures logged from 2003 to 2009 for 6 Canadian urology programs that voluntarily participated. All data were third-party blinded for individual program and resident identification prior to its release to the authors. A listing of the 22 pediatric urological procedures from the RCPSC OTR in Urology (Table 1) was compiled and the number of 


\begin{tabular}{lcc}
\hline \multicolumn{3}{l}{ Table 1. 2009 RCPSC objectives of training for pediatric urology procedures } \\
\hline A List Procedures & B List Procedures & C List Procedures \\
\hline Endoscopic & Endoscopic & Open \\
\hline TUR/incision of ureterocele & Resection of posterior urethral valves & Correction of proximal hypospadias and epispadias \\
Endoscopic stone management & Open & Surgical reconstruction for exstrophy \\
Endoscopic injection for VUR & Vesicostomy & Renal transplantation \\
Open & Augmentation cystoplasty \\
Circumcsion & Continent urinary reservoir \\
Meatotomy/meatoplasty & Correction of mid and distal shaft hypospadias & \\
Meatal repair for glanular & Laparoscopic \\
hypospadias & Orchidopexy/orchiectomy for abdominal testis \\
Pediatric Indirect hernia repair & Pyeloplasty \\
Hydrocelectomy & \\
Pediatric indirect hernia repair & \\
Repair of testicular torsion & \\
Uretero-neocystotomy & \\
Pyleoplasty for UPJ obstruction & \\
\hline VUR: vesicoureteral reflux; TUR: transurethral resection; UPJ: ureteropelvic junction. \\
\hline
\end{tabular}

cases logged by residents in the participating programs in the T-Res database for each of these procedures was tabulated. Data were collected in an Excel (Microsoft Corp., Redmond, WA) spreadsheet. The average number of cases the residents performed per year for each of the procedures was then calculated.

The objectives from the RCSPC are determined and categorized by the urology special committee members, a joint committee of the Royal College and the Canadian Urological Association. As outlined by the RCPSC, category-A procedures are those in which "all residents must be competent to independently perform ..., be able to manage a patient prior to, during and after ... [and] be able to describe the management of the common complications." Category-B procedures "are those that the resident will know how to do, including indications. ... the resident may not have actually done one of these procedures independently during the residency training program." Category-C procedures "are those for which the resident will be able to describe the principles of the procedure, indications for referral for the procedure and particular perioperative problems that might be encountered." ${ }^{3}$

\section{Results}

A total of 48 residents submitted data to T-Res from the 6 participating programs. The number of residents submitting data from a given program ranged from 3 to 15 . For each of the category A, B and C procedures, the average number of cases performed per year by a given resident was calculated for each program (Table 2). Of the A-list procedures, Canadian urology residents (PGY 1-5) from the 6 participating programs participated in an annual average of 53 hypospadias repairs, 30 orchidopexies for inguinal testes, 26 circumcisions, 7 hernia/hydrocele repairs, 7 pyeloplasties, 7 ureteral reimplants, 6 endoscopic injections for vesicoureteral reflux (VUR), 3 meatoplasties/meatotomies, 1 transurethral incision of ureterocele, 2 endoscopic procedures for stone management, and 1 transurethral incision of ureterocele, during the years in question. Of the B-list procedures, residents participated in an annual average of 1 transurethral resection (TUR) of a posterior urethral valve, 3 continent diversions, 2 augmentation cystoplasties, and 1 vesicostomy. Of the data available for the $\mathrm{C}$-list procedures, residents participated in an annual average of less than 1 exstrophy repair and less than 1 pediatric renal transplant.

\section{Discussion}

There is no consensus on the number of cases that need to be performed to achieve an adequate level of competency to carry out a given operation independently.

Moreover, operative competence itself encompasses more than merely the technical ability to perform a procedure. As noted by Grober and Jewett, it "captures a trainee's cognitive, technical and communicative skills, as well as confidence, experience, poise, professionalism and judgment. ${ }^{\prime \prime}$ Nevertheless, our study helps to frame the conversation on competence in performing core pediatric urologic procedures by enumerating the exposure to these procedures during residency.

The Urology Specialty Committee revised the RCPSC objectives in 2009 with notable changes to the category A and B lists of procedures. Important additions to the A-list procedures included endoscopic injection for VUR and TUR/incision of ureterocele. Augmentation cystoplasty and continent diversion were also moved from the A-category to the B-category, while renal transplantation was moved 


\begin{tabular}{|c|c|c|c|c|c|c|c|}
\hline & Program 1 & Program 2 & Program 3 & Program 4 & Program 5 & Program 6 & Sum \\
\hline A Procedures & Avg/year & Avg/year & Avg/year & Avg/year & Avg/year & Avg/year & \\
\hline Circumcision & 66.90 & 31.00 & 10.88 & 23.04 & 12.17 & 11.10 & 26 \\
\hline Hernia repair & 25.14 & 5.14 & 2.50 & 3.23 & 3.25 & 0.83 & 7 \\
\hline Hydrocelectomy & 14.32 & 2.50 & 3.25 & 10.42 & 3.67 & 9.17 & 7 \\
\hline Orchidopexy for inguinal testis & 77.28 & 24.20 & 10.38 & 30.03 & 14.00 & 24.67 & 30 \\
\hline Meatoplasty/meatotomy & 9.87 & 4.29 & 1.00 & 1.85 & 0.00 & 0.83 & 3 \\
\hline Hypospadias repair & 54.04 & 33.21 & 6.75 & 10.85 & 14.17 & 24.73 & 53 \\
\hline Pyeloplasty & 12.13 & 4.29 & 3.75 & 4.62 & 3.58 & 11.40 & 7 \\
\hline Ureteral reimplant & 17.07 & 11.29 & 3.00 & 7.15 & 0.75 & 5.57 & 7 \\
\hline Transurethral ureterocele incision & 2.67 & 0.86 & 1.75 & 0.00 & 0.00 & 2.33 & 1 \\
\hline Endoscopic injection for VUR & 5.81 & 1.29 & 12.62 & 0.92 & 1.00 & 12.83 & 6 \\
\hline Endoscopic stone procedure & 3.25 & 1.71 & 0.50 & 1.15 & 0.83 & 1.93 & 2 \\
\hline B Procedures & Avg/year & Avg/year & Avg/year & Avg/year & Avg/year & Avg/year & \\
\hline Vesicostomy & 2.67 & 1.07 & 0.00 & 0.00 & 1.67 & 0.00 & 1 \\
\hline TUR posterior urethral valves & 2.33 & 0.43 & 0.25 & 0.69 & 0.00 & 5.00 & 1 \\
\hline Continent urinary reservoir & 7.07 & 6.07 & 0.00 & 0.92 & 2.50 & 0.00 & 3 \\
\hline Augmentation cystoplasty & 7.13 & 0.86 & 0.00 & 1.85 & 0.83 & 0.00 & 2 \\
\hline C Procedures & Avg/year & Avg/year & Avg/year & Avg/year & Avg/year & Avg/year & \\
\hline Exstrophy & 2.43 & 2.14 & 0.00 & 0.00 & 0.00 & 0.00 & 1 \\
\hline Renal transplant & 4.60 & 1.29 & 0.00 & 0.00 & 0.00 & 0.00 & 1 \\
\hline
\end{tabular}

from the B- to the C-category. This iterative reassessment process of the OTR by the Specialty Committee (which was most recently repeated in June 2013) is an important component of keeping our specialty current with contemporary advances in technology, practice patterns and demographics. However, the OTR are based on expert opinion, which unfortunately is the lowest level of evidence in the hierarchy of evidence-based medicine. ${ }^{6}$ Until such time that we have mandatory implementation of national e-portfolios to document trainee exposure and the use of valid measures of surgical competence, we have no other resource than expert opinion to craft the OTR for our trainees in Canadian urology. With the impeding implementation of competence-based medical education (CBME) from the RCPSC, we should see a gradual shift towards the use of more objective measures of trainee experience and competence. ${ }^{7}$

In a previous study from 2008, Mickelson and colleagues conducted a survey of urology residency program directors, senior urology residents and members of Pediatric Urologists of Canada (PUC) from all 12 Canadian training programs. ${ }^{4}$ The focus was on 23 pediatric urological procedures the 3 study groups perceived urology residents would be competent to perform upon completion of residency. The biggest discrepancies among respondents to their survey surrounded 4 procedures in category-A (infant pyeloplasty, ureteral reimplant, augmentation cystoplasty, infant heminephrectomy) and 3 in category-B (veiscostomy, distal shaft hypospadias, TUR of posterior urethral valves) procedures. Both residents and PUC members were less likely, than their program direc- tors, to perceive residents technically competent to perform these 7 procedures.

Our study reveals that, on average, residents are participating in 7 pyeloplasties, 7 ureteral reimplants, 2 augmentation cystoplasties, and 2 heminephrectomies per year. This is a relatively small number of procedures compared with other category-A procedures, such as circumcision and orchidopexy for inguinal testis where residents perform 26 and 30 per year, respectively (Table 2 ). With respect to the B-category, our data show that residents participated in 1 vesicostomy and 1 TUR of posterior urethral valve per year, which also seems to be a relatively small volume of cases. This would appear to explain why many trainees and their instructors did not deem residents competent to perform these procedures independently upon graduation.

Given these results, the revisions to the objectives set out by the RCPSC in 2009 seem appropriate. There have been significant changes in the trends for management for common pediatric urologic conditions as our understanding of these conditions has evolved. Surgical correction with ureteral reimplation for VUR, for example, is no longer considered the only standard of care. ${ }^{8}$ This once common procedure is now often supplanted by endoscopic injection therapy, which is reflected in the current set of objectives.

Complex procedures, such as bladder augmention, continent diversion, infant pyeloplasty and heminephrectomy, require a more specialized skill-set than that which may conceivably be developed during residency. Accordingly, augmentation cystoplasty and continent diversion have been 
moved to the B-category, while infant hemi-nephrectomy no longer appears as an objective. Our results support the categorization of these procedures as better suited to the $\mathrm{B}$ - rather than the A-list given the relatively small number of these cases residents are exposed to during their training.

Our results show considerable variability in operative exposure among the participating programs in the category $\mathrm{A}, \mathrm{B}$ and $\mathrm{C}$ procedures. As discussed above, there is no defined number of cases that need to be performed to achieve competency for a given operation, but graduates of programs with a greater volume of cases may be better equipped to independently perform the A-category procedures.

There are limitations to our study. The raw data obtained from the T-Res database are only a sampling from $50 \%$ of Canadian programs that voluntarily participated in data gathering during the study period. The other Canadian programs did not submit their T-Res data for inclusion in the study, which limits our ability to generalize our results to the national pediatric urology training experience.

In addition, for some procedures, the data are incompletely classified. For example, we were unable to ascertain the number of distal shaft hypospadias repairs in which residents participated. Cases were recorded as "hypospadias repairs" without detailing if the repairs were at the level of the glans penis, distal shaft or proximal shaft. This distinction is important as balanic hypospadias repairs are considered to be category-A procedures, while distal shaft and proximal shaft repairs are considered to be in the B- and C-category, respectively. That being said, residents are participating in an average of 53 hypospadias repairs in general per year according to our study, which appears to be a robust caseload. Presumably, most of these cases are glanular or distal shaft repairs. ${ }^{9}$ In addition, the extent of resident involvement for the cases logged is also incompletely classified based on the available data. Our results convey the number of cases in which a resident is involved, but the degree of resident participation may range from observer to primary operator. This is an important consideration when evaluating how competent a resident is to perform a procedure independently, the benchmark for determining whether or a not a category-A objective has been met.

Similarly, without operative details, we were not able to determine if testes were palpable in the orchidopexies recorded in the database. Non-palpable orchidopxies and laparoscopy for non-palpable testes are category-B procedures, while palpable orchidopexies are category-A procedures. This also precluded us from establishing the exposure to repairs of testicular torsion as the clinical details were not available for the orchidopexies and orchiectomies performed.

Although benchmark numbers for index cases have not been defined, the authors clearly demonstrate that there is a considerable variability of pediatric urology operative exposure across training programs, particularly in category-A procedures. This is arguably where we should see the most concordance.

\section{Conclusion}

The extent to which pediatric urological procedures should be performed by general urologists is contentious. Considering that Canada's population is widely dispersed over a large geographic area, it will be necessary for general urologists to continue to provide primary and secondary care for many pediatric conditions to provide adequate and timely access for families situated far from tertiary centres. The RCPSC objectives set out by the specialty committee are a useful framework to guide graduating residents on which procedures they might reasonably perform once they enter practice. Ongoing revisions to these objectives, which reflect changing trends in the management of core pediatric urology procedures, are supported by our study based on the number of cases in which residents participate. Improvements in the assessment of trainee surgical experience and competence as it relates to the objectives of training in pediatric urology are required as we migrate towards a competency-based model of postgraduate medical education.

Competing interests: Dr. Gustafson and Dr. MacNeily declare no competing financial or personal interests.

This paper has been peer-reviewed.

\section{References}

1. Rushton HG. Subspecialty certification in pediatric urology. J Urol 2006;173:1845-6. http://dx.doi. org/10.1097/01.ju.0000163662.01660.df

2. The Society for Pediatric Urology. Pediatric subspecialty certification. www.spuonline.org/PediatricSubspecialty-Cerrification/. Accessed September 15, 2014.

3. The Royal College of Physicians and Surgeons of Canada. Objectives of training in urology. http://www. royalcollege.ca. Accessed September 14, 2014.

4. Mickelson JJ, MacNeily AE, Samarasekera D, et al. Competence in pediatric urology upon graduation from residency: Perceptions of residents, program directors and pediatric urologists. Can Urol Assoc $\mathrm{J}$ 2008;2:205-10.

5. Grober ED, Jewett MAS. The concept and trajectory of "operative competence" in surgical training. Can J Surg 2006; 49:239-40.

6. Shekelle PG, Woolf SH, Eccles M, et al. Developing clinical guidelines. West J Med 1999;170:348-51.

7. Iobst WF, Sherbino J, Cate OT, et al. Competency-based medical education. Medical Teacher 2010;32:651-6. http://dx.doi.org/10.3109/0142159X.2010.500709

8. Montini G, Tullus K, Hewitt I. Febrile urinary tract infections in children. N Engl J Med 2011;365:3. http://dx.doi.org/10.1056/NEJMra1007755

9. Snodgrass WT. Hypospadias. In: Campbell-Walsh Urology. 10th ed. Phildelpia, PA: Elsevier; 2012:350336. http://dx.doi.org/10.1016/B978-1-4160-6911-9.00130-4

Correspondence: Dr. Paul Gustafson, Department of Urologic Sciences, University of British Columbia, Vancouver, BC; pgustafson1@gmail.com 